\title{
Yang-Mills-Higgs-Kibble 系统的静态解
}

\author{
S. Deser* C. J. Isham \\ （英国帝国科学技术学院 Blackett 实验室）
}

\section{一、引言}

无质量的杨一米尔斯场在提供静态有限能量解上和与它相应的 Abel 场不同. 这个情况当 且仅当时空为 5 维时发生 ${ }^{[1,2]}$, 这时这些解对应于等价的 4 维欧氏空间中存在着瞬子解 ${ }^{[3]}$. 在 最近的工作中, 胡和生 ${ }^{[1]}$ 考虑了有质量 Yang-Mills 场中同样的问题, 特别证明了在 5 维时不存 在静态解. 虽然, 如所周知 ${ }^{[5,6]}$, 从一个环路的水平出发, 在质量趋向于零的极限时存在着不连 续性, 而她的结果是我们认识到存在着一个经典的不连续性（即解仅当质量 $m$ 为 0 时才存在） 的第一个明确的例子.

当然瞬子仅当 $m=0$ 时才存在. 从物理上来看是不奇怪的, 因为它们的无标度的性质不 为 4 维欧氏的有质量模型所享有. 然而, 这个不连续性引起了这样的问题: 比起具有显式质 量项的理论来, 用 Higgs-Kibble 机制把质量 “较软的”导入是否将导致一个比较光滑的极限. 我 们建议在任意维数下考察这样的模型, 并把结果与无质量的及“硬”质量情形下的结果相比较. 特别, 我们将看到, 在 5 维时, 不连续性继续存在一一这最终可归因于 Higgs 势的 $\phi^{2}$ 部分 (尽 管不是 $\boldsymbol{\phi}^{4}$ 部分) 的内在的标度破缺。

\section{二、軤态系统的条件}

让我们先开始回顾一下决定静态解存在的一般条件 ${ }^{[2]}$. 对任何封闭系统, 能量动量张量 $T_{\nu}^{\mu}$ 适合恒等式

$$
\partial_{\mu}\left(T_{\nu}^{\mu} x^{\nu}\right)-T_{\mu}^{\mu}=0,
$$

因而如体系为静态时, 所有物理量的时间导数均为 0 , 则得 $\partial_{0}\left(T_{v}^{0} x^{\nu}\right)=T_{0}^{0}$, 因而

$$
-T_{0}^{0}+T_{\mu}^{\mu} \equiv T_{i}^{i}=\partial_{i}\left(T_{v}^{i} x^{\nu}\right)
$$

(或者, $\partial_{i}\left(T_{j}^{i} x^{i}\right)-T_{i}^{i}=0$, 这是因为 $\left.\partial_{0} T_{j}^{0}=0\right)$. 注意: 我们对时空指标采用希腊字母, 对 空间维数采用拉丁字母, 且所论的总维数为 $D$ 的时空具符号 $(++++\cdots-)$. 在 $(D-1)$ 维 空间上积分 (2), 再利用能量有限时(2)的右边的散度必须足够快地为 0 的事实,得出

$$
\int T_{i}^{i} d^{D-1} x=0,
$$

方程(3)表明一个静态系统的自应力必为 0 .

为了对有兴趣的情形计算 $T_{i}^{i}$, 我们将对具 Higgs 标量多分量态 $\phi$ 及势 $V(\phi)$ 的有质量 的 Yang-Mills 系统采用作用量

*此研究由 NSF Grant PHY 78-09644-A01 部分资助. 永久地址为 Brandeis University。 


$$
I=\int\left\{-\frac{1}{4} F_{\mu \nu} F^{\mu \nu}-\frac{m^{2}}{2} A_{\mu} A^{\mu}+\left[-\frac{1}{2} \nabla_{\mu} \phi \nabla^{\mu} \phi-V(\phi)\right]\right\}
$$

(此处我们已去掉了内部指标), 因而也可算出相伴随的应力张量. 相应的对称化的应力张量 为

$$
\begin{aligned}
T^{\mu \nu} & =F_{\alpha}^{\mu} F^{\nu \alpha}+m^{2} A^{\mu} A^{\nu}+\nabla^{\mu} \phi \nabla^{\nu} \phi \\
& -g^{\mu \nu}\left[\frac{1}{4} F_{\alpha \beta} F^{\alpha \beta}+\frac{m^{2}}{2} A_{\alpha} A^{\alpha}+\frac{1}{2} \nabla_{\alpha} \phi \nabla^{\alpha} \phi+V(\phi)\right] .
\end{aligned}
$$

借助于 $E^{i} \equiv F^{o i}$ ，空间部分的迹为

$$
\begin{aligned}
T_{i}^{i}= & \frac{1}{2}(D-3) E^{i} E^{i}-\frac{1}{4}(D-5) F^{i j} F^{i j}-\frac{m^{2}}{2}(D-3) A_{i} A_{i} \\
& +\frac{m^{2}}{2}(D-1) A_{0}^{2}-\frac{1}{2}(D-3) \nabla_{i} \phi \nabla_{i} \phi-(D-1) V(\phi)+\frac{m^{2}}{2}(D-1)\left(\nabla_{0} \phi\right)^{2},
\end{aligned}
$$

这里 $\delta_{i}^{i}=D-1$.

原先的 Yang-Mills 情形相应于 $m=\phi=0$.(3)式导致文献[2]中结果:除了 $D=5$ (即 4 维欧氏空间)外,不存在解. 对于“硬的”有质量的系统 $m \neq 0, \phi=0$, (3) 导致文献[4] 中的 结果: 对 $D \neq 4$, 无静态解, 所以特别对 $D=5$ 无静态解, 但对 $D=4$, 只找到了较弱的条件

$$
\int\left\{\frac{1}{4} F^{i j} F^{i j}-\frac{m^{2}}{2} A_{i} A_{i}\right\} d^{3} x=0 .
$$

这个条件并不妨碍静态解的存在, 但也不能说从它一定能推出静态解的存在,因为自应力条件 并没有用尽了包含在场方程中的信息. 于是, (3) 式只有在它本身禁止了解的存在时,才是决 定性的条件. 例如在有质量的 Abel 情形, (7)式仍然成立,但我们从场方程中能直接看出

$$
0=\int \boldsymbol{A} \cdot\left(-\nabla \times \boldsymbol{B}+m^{2} \boldsymbol{A}\right) d^{3} x=\int\left(\boldsymbol{B} \cdot \boldsymbol{B}+m^{2} \boldsymbol{A} \cdot \boldsymbol{A}\right) d^{3} x,
$$

于是仅有的解是 $\boldsymbol{B}=\boldsymbol{A}=0$. 在任何情况下, 当 $m \rightarrow 0$ 时趋向于 0 的具质量的解的存在并 不表示不连续性,但在相反的情形,即仅当 $m=0$ 时才存在解,才表示不连续性.

我们用关于静态系统的定义的某些评论来结束本节. 对非规范场 $(m \neq 0)$, 向量势 $A_{\mu}$ 本 身是可观察量, 且静态意味着 $\partial_{0} A_{\mu}=0$, 而对规范场的情形 $(m=0)$, 静态条件严格地说仅 应用于如 $F_{\mu \nu} \cdot F^{\mu \nu}$ 或 $E \cdot B$ 等等的规范不变量.显然人们能通过非实质的规范选择导人 $F_{\mu \nu}$ 或同位旋向量 Higgs 场 $\phi$ 关于时间的显式的依赖性, 虽然这总是能够 (而且应当) 避免的. 一 个更加进一步的要求是向量势本身为静态的, 例如 $\partial_{0} A_{i}=0$. 下列事实并非先验显然的, 即所 有具静态不变量的解能允许一个规范选择, 使得 $\partial_{0} A_{i}$ 为 0 , 虽然这看上去好象是可能的 (实际 上这是可以证明的一译者注). 从这个选择可以得出: 电场为 0 , 这是因为

$$
F_{o i}=\partial_{0} A_{i}-\partial_{i} A_{0}-A_{i} \times A_{0} \rightarrow-\nabla_{i} A_{0},
$$

此处 $\nabla_{i} \equiv \partial_{i}+A_{i} x$ 是作用在同位旋向量上的共变导数. 当用 $A_{0}$ 乘 Gauss 方程 $\nabla_{i} E_{i}=0$, 积分后可得出 $\nabla_{i} A_{0}=E_{i}=0$. 在有质量的情形, $\nabla_{i} E_{i}=m^{2} A_{0}$. 由此过程知道 $E_{i}$ 及 $A_{0}$ 两 者均为 0 . 最后在 Higgs 情形下, 因为 $\nabla_{0} \phi=A_{0} \times \phi$, 所以由 $\nabla_{i} E_{i}=\frac{\delta}{\delta A_{0}}\left(A_{0} \times \phi\right)^{2}$ 证明了 $E_{i}$ 及 $\nabla_{0} \phi$ 两者均为 0 . 从 $A_{0} \times \phi$ 为 0 , 我们知道在同位旋空间中 $A_{0}$ 平行于 $\phi$. 更一般地, 只要某个同位旋向量平行于 $\phi$, 则可得出 (至少对 $S U_{2}$ 及其他单纯的内部群)它指向一个固定 的同位旋方向, 这是因为我们能在任意点用 $\phi(x)$ 定义 (譬如说是) 第 3 轴方向. 在下面我们 将利用这个附注。 


\section{三、Yang-Mills-Higgs-Kibble 系统}

现在我们来讨论把 Yang-Mills-Higgs 系统中显式质量项丢掉后,(6)所蕴含的条件. 为此, 我们首先必须讨论 Higgs 势 $V(\phi)$ 的性质. 它具有形式

$$
V(\phi)=-\frac{\mu^{2}}{2} \phi^{2}+\frac{\lambda}{4} \phi^{4} \equiv \frac{\lambda}{4}\left(\phi^{2}-\frac{\mu^{2}}{\lambda}\right)^{2}+\frac{\mu^{2}}{4 \lambda},
$$

常数项没有物理意义, 此处应当丢掉, 以避免 $T_{\mu \nu}$ 中的一个不合逻辑的“宇宙”项, 并保证我们 要寻找的解的能量的有限性. 我们仅对关于 Higgs 点 $a^{2}=\frac{\mu^{2}}{\lambda}$ 处的邀发感兴趣, 它给向量予 质量. 但是注意: 与硬质量的情形相比较, 只有与惯常选择的 $\phi$ 方向相正交的同位旋分量才 获得了质量, 而与 $\phi$ 方向相平行的分量仍然没有质量. 我们可以着手于 (3), (6), 取 $V(\phi)$ 为 非负的, 且 $V(\phi)=0$ 能推出常值解 $\phi^{2}=\frac{\mu^{2}}{\lambda}$. 根据在前节末的讨论, $E^{i} E^{i}$ 及 $\left(\nabla_{0} \phi\right)^{2}$ 项亦可 丢掉. 我们现考虑 $D$ 的各种值.

\section{a) $D>5$}

方程 (6) 是负项之和, 因此它的每一项必为 0 , 于是得出 $F_{i j}=\nabla_{i} \phi=V(\phi)=0$, 此处 $V(\phi)=0$ 意味着 $\phi$ 为常值, 因而不存在本质上非平凡的解, 使得它与 $m=0$ 及 $m \neq 0(\phi=$ 0) 模型相一致(实际上, $\nabla_{i} \phi=0$ 能推出 $A_{i} \times \phi=0$, 所以 $A_{i}$ 是在一个固定的同位旋方向 上, 这意味着 $A_{i} \times A_{i}=0$, 因而 $F_{i j}=\partial_{i} A_{j}-\partial_{i} A_{i}=0$, 这表明 $A_{i}$ 是一个纯粹的 Abel 规 范).

b) $D=5$

此处(6)蕴含 $\nabla_{i} \phi=V(\phi)=0$, 看上去它好像允许非平凡的纯 Yang-Mills 解. 但是情 况并非如此, 这是因为 $\phi^{2}=\frac{\mu^{2}}{\lambda} \neq 0$ 及 $\nabla_{i} \phi=0$ 能推出 $A_{i}$ 是在一个固定的同位旋方向上. 但 从这个 Abel 的情形出发, 我们可以如 Maxwell 情形那样, 推出 (尽管不直接从 (3) 及 (6)) $\nabla_{i} F^{i j}=\partial_{i} F^{i j}=0$, 于是 $F_{i j}=\partial_{i} A_{i}-\partial_{i} A_{i}=0$. 由此我们看到, 如同在硬质量情形那样, 存 在着不连续性. 显然这是由于损失了标度, 因为当我们令 $\mu^{2} \rightarrow 0$ 及 $\phi^{2}$ 为 0 时, 我们不再能从 $\nabla_{i} \phi=0$ 推出关于 $A_{i}$ 的任何东西. 因此不连续性已被有效地转移到新的质量参数 $\mu^{2}$ 上去 了.

c) $D=4$

我们有 $\int\left(\frac{1}{4} F_{i j} F_{i j}-\frac{1}{2} \nabla_{i} \phi \nabla_{i} \phi-3 V(\phi)\right) d^{3} x=0$, 因为被积项不是一个正的量, 所以 不能告诉我们任何东西. 对 $m \neq 0(\phi=0)$ 时情形也如此, 但在 $m=0$ 的情形则不允许解. 事实上我们知道存在着由 Higgs 场所提供的非奇异解 ${ }^{[9]}$, 所以由于物质源的存在, 引起了一个 真正的物理的差异.

d) $D=3$

我们有非决定性的限制 $\int\left(\frac{1}{2} F_{i i} F_{i j}-2 V(\phi)\right) d^{2} x=0$, 这与 $m=0$ 及 $m \neq 0(\phi=0)$ 时 的情形不同, 那时它们都没有解. 充分利用二维欧氏 Yang-Mills 模型的特殊性质, 人们可能通 过研究场方程直接地解决解的存在性的问题, 但我们还没有做到(虽然我们注意到, 如 $V=0$, 则无解). 
存在着一个有趣的值得关心之点. 在 $D=3$ 的情形, 不存在 $m \neq 0$ 的解是比较惊奇的. 考虑一个作用量, 它是 Yang-Mills 及 $\sigma$ 模型部分的和. 前者无静态解, 但众所周知 ${ }^{[7]}$, 后者有 静态解; 这是显然的, 因为对于一个无质量的, 但有自相作用的标量场, (6)中的 $(D-3)$ 因子 去掉了动力学部分 $f_{i i}(\phi) \partial_{\mu} \phi^{i} \partial_{\mu} \phi^{j}$. 另一方面, 也已经知道 ${ }^{[6,8]}$, 有质量的 Yang-Mills 场的纵向 模式本质上是 $\sigma$-模型的形式, 但是它与横向模式相互作用着. 于是这个相互作用明显地把其 他的可允许作为 $\sigma$-模型部分的解去掉了.

\section{四、结论}

我们已经将无质量的 Yang-Mills 场的静态解的性质与具有质量的两种可能的推广的静态 解的性质作了比较; 一种推广是 “硬” 型的, 另一种推广通过 Higgs-Kibble 机制, 它把质量给予 同位旋三个分量中的二个分量 (对任意的紧致单纯内部对称群, 存在着明显的推广). 所有模 型对 $D>5$ 均一致, 但对 $D=5$, 每一种形式都具有与质量标度的存在相伴随的一种不连续 性.（仅仅)对 $D=4$, Higgs 模型允许一个静态解, 而仅在 $D=3$ 时, Higgs 理论中的解没有 立即被排除.

显然以一般的方法,利用例如 Stueckelberg 分解的某些类型 ${ }^{[6]}$ (在 Abel 的情形下,这种分解 是有用的),对 “硬” 理论的无质量极限进行探讨是极有兴趣的. 我们希望在别处回到这个问 题.

致谢: S. Deser 对提供高级访问基金的科学研究委员会及帝国学院理论组的好客表示感谢.

\section{考文献}

[1] Coleman, S., Erice Lectures, Appendix II, 1975.

[ 2 ] Deser, s., Phys. Letts., 64B (1976), 463.

['3] Belavin, A., Polyakov, A., Schwarz, A. \& Tyupkin, X., Phys. Letts., 59 B (1975), 85.

[ 4] Hu Hesheng (胡和生), 科学通报,25(1980),6:241.

[ 5 ] Veltman, M., Nucl. Phys., B21 (1970), 288; Van Dam II. \& Veltman, M., ibid., B22 (1970), 397.

[6] Boulware, D., Ann. Phys., 56 (1970), 140.

['7] Belavin, A. \& Polyakov, A., JETP Letts. (Bov. Phys.), 22 (1975;, 245;

Duff, M. J. \& Isham, C. J., Nucl. Phys., B114 (1976), 29;

Honerkamp, J.. Patani, A., Sehlindwein, M. \& Shafi, Q., Nuovo Cim. Letts., 15 (1976), 97.

[ 8 ] Deser, S., Phys. Rev., 187 (1969), 1931.

[' 9 ] 't Hooft, G., Nucl. Phys., B79 (1974). 276;

Polyakov, A. M., JETP Lett., 20 (1974), 194;

Prasad, M. K. \& Sommerfield, C., Phys. Rev. Lett., 35 (1975), 760.

[胡和生 译] 